conflict with physical principles. Energy is absorbed by the shell from the magnetic field and, as with all systems having resonance properties, more energy is absorbed when the period is near one of the natural periods of the system.

It is clear from the above that our general concoption of the ionosphere acting as a screen from magnetic fluctuations originating outside it ${ }^{1}$ will need considerable modification. It will still shield the Earth from the most rapid fluctuations occurring outside it, but for fluctuations having periods of the order of a few minutes or tens of minutes, there may be no shielding at all, but even an amplification of the outside field. These conclusions may of course be somewhat modified when the low ionization on the night side of the Earth is taken into account. Our detailed calculations will be published elsewhere.

Department of Mathematics,

$$
\text { A. T. Price }
$$

University of Exeter.

Department of Mathomatics,

$$
\text { G. A. J. Ferris }
$$

University of Leeds.

Ashour, A. A., and Price, A. T., Proc. Roy. Soc., A, 195, 198 (1948).

\section{Induction of Electric Currents in a Uniform Anisotropic Ionosphere}

THE problem of the induction of electric currents in a non-uniform ionosphere is of interest in geomagnetism. It was first studied by Ashour and Price ${ }^{1}$ to determine the effects to be expected from the greater conductivity of the ionosphere over the sunlit hemisphere. Some time ago we considered the electric currents induced in a uniform anisotropic ionosphere (thus ignoring the difference in conductivity between the day and night hemispheres). We found that the anisotropy modifies only that part of the inducing field which is asymmetrical with respect to the geomagnotic axis, and that this asymmetrical part rotates about the geomagnetic axis from wost to east at a rate depending on the Hall conductivity.

Treating the ionosphere as a spherical current sheet of radius $a$, and assuming that the current arises mainly from the flow of electrons, we find that the resistivity tensor is of the form:

$$
K=\frac{1}{\bar{\sigma}_{0}}\left\{\begin{array}{c}
1, \beta \cos \psi \\
-\beta \cos \psi, 1
\end{array}\right\}
$$

where $\bar{\sigma}_{0}$ is the integrated normal conductivity of the sheet, $\psi$ is the inclination of the local magnetic field to the geomagnetic axis, and $\beta$ is the ratio of the Hall conductivity to the ordinary conductivity at the pole.

The magnetic potentials, $\Omega^{(e)}$ and $\Omega^{(i)}$, due to the external inducing field and induced currents, respoctively, are solutions of Laplace's equation and the induced field is determined from the conditions that, in passing through the current sheet, the normal resolute of the magnetic field and of the tangential eomponent of the electric field are both continuous. Denoting by $(r, \theta, \varphi)$ the spherical polar co-ordinates of a field point, then assuming that an external inducing ficld derived from a potential:

$$
\Omega^{(e)}=a \Sigma E_{n}^{m}(t)\left(\frac{r}{n}\right)^{n} P_{n}^{m}(\cos \theta) \exp i m \varphi
$$

is suddenly set up outside the ionosphere, we find that the total field inside the sheet aftor time $t$ is derived from the potential:

$$
\begin{aligned}
& \Omega=\Omega^{(e)}+\Omega^{(i)}= \\
& a \Sigma E_{n}^{m}\left\{1-\exp \left[-\left(p_{n}^{m}+i q_{n}^{m}\right) t\right]\right\} \\
& \quad\left(\frac{r}{a}\right)^{n} P_{n}^{m}(\cos \theta) \exp i m \varphi
\end{aligned}
$$

where:

$$
p_{n}^{m}=\frac{(2 n+1)}{4 \pi a \bar{\sigma}_{0}} \quad q_{n}^{m}=\frac{(2 n+1) m \beta}{4 \pi a(n+1) n} \frac{1}{\bar{\sigma}_{0}}
$$

We note that $q_{n}^{m}=0$ if $\beta=0$ or $m=0$; in the former case the sheet is isotropic and in the latter case the field is axially symmetric about the geomagnetic axis. In this case, the sheet clearly behaves as if it were isotropic.

The presence of $q_{n}^{m}$ in the imaginary part of the time foctor shows first that, as was to be expected on general grounds, the Hall conductivity does not affect the rate of decay of the currents and, secondly, that the asymmotric part of the induced field rotates from west to east about the geomagnetic axis. Price and Ferris (private communication) have shown that this result is also true for a more general model of the ionosphere, and that the rotation of the asymmetrical part of the field seems likely to be of importance in causing a resonance effect in the ionosphere.

As a numerical illustration of the orders of magnitude involved, we have taken the case when $n=1$, $m=1$ (corresponding to a uniform field), so that:

$$
q_{1}^{1}=\frac{3 \beta}{8 \pi a \bar{\sigma}_{0}}
$$

Then, taking Martyn's value of the integrated conductivity $\bar{\sigma}_{0}\left(=5 \times 10^{-8}\right.$ e.m.u. $)$ and $\beta=7$, and putting $a=6.5 \times 10^{8} \mathrm{~cm}$ (the radius of the Earth) we find that the period of rotation of the asymmetric part of the field induced by setting up of a uniform external field normal to the geomagnetic axis is about $4 \mathrm{~min}$.

\section{University of Cairo.}

$$
\text { A. A. Ashour }
$$

Queen Mary College,

$$
\text { v. C. A. Ferraro }
$$

London, E.1.

${ }^{1}$ Ashour, A. A., and Price, A. T., Proc. Roy. Soc. A, 195, 198 (1948).

\section{ENGINEERING}

\section{Permanent Insulating Duct Walls for Magnetohydrodynamic Power Generation}

Most of the reported experimental work on magnetohydrodynamic generation using hydrocarbon and other flames has been of limited duration owing to the difficulties in providing duct and electrode materials. These must withstand very high temperature (around $2,500^{\circ} \mathrm{C}$ ), high velocity (near sonic), corrosive (a percentage or more of potassium seeding) gas conditions.

A possible method of obtaining permanent electrodes for magnetohydrodynamic power generation under these conditions has already been proposed ${ }^{1}$, but the problem of providing electrically insulating duct walls remains. High-temperature materials such as magnesia have boen tried with limited success ${ }^{2}$; but they can scarcely be called permanent as yet.

At the temperatures envisaged, the products of combustion contain appreciable amounts of atomic oxygen and hydrogen in equilibrium, as well as other free radicals, and are therefore highly corrosive. All 